\title{
Psychogenic medicine
}

\section{Non-organic visual loss}

\author{
S Beatty
}

\begin{abstract}
Summary
Visual complaints without a physical basis are not uncommon presentations to the general physician, the neurologist, or the ophthalmologist. These alleged visual disturbances may be psychogenic or feigned. The diagnosis is made when all possible contributory pathology of the visual system is excluded, and reassurance remains the cornerstone of management.
\end{abstract}

Keywords: blindness; electroretinography; evoked potentials; psychogenic illness

Techniques to test the validity of an alleged profound reduction in vision

- menace reflex: an intact menace reflex indicates some degree of crude vision

- mirror test: rotate a mirror before the 'blind' eye and observe for pursuit movements

- optokinetic nystagmus: if optokinetic nystagmus can be elicited, a Snellen acuity of $6 / 60$ or better is confirmed

- simple tests of proprioception: a truly non-seeing subject will be able to perform these tasks with relative ease; the malingerer, believing these tasks to be vision-dependent, fails

Manchester Royal Eye Hospital, Oxford Road, Manchester M13 9WH, UK S Beatty

Accepted 16 November 1998
Visual complaints without evidence of contributory ocular or non-ocular pathology are not infrequent presentations to the general Accident and Emergency department, and are said to account for approximately $1 \%$ of visual problems seen by the ophthalmologist. ${ }^{1}$ The wide spectrum of terminology used to describe visual disturbance without a physical basis, which includes 'functional', 'hysterical' (amblyopia), 'conversion disorder', 'neurotic', 'supratentorial', ' and 'psychosomatic', has resulted in considerable confusion. In this review we use the term non-organic visual loss (NOVL) to describe any visual disturbance where there is no detectable dysfunction of structures between cornea and occipital cortex.

NOVL may be psychogenic, or the result of malingering. Psychogenic visual complaints result from a disturbance of higher cortical structures occupied with visual awareness, and patients with this form of NOVL do experience but do not control their visual symptoms. The malingerer, on the other hand, deliberately feigns visual loss for secondary gain. The term 'malingering' is in many ways a moral accusation rather than a clinical diagnosis, and consequently should only be used with extreme caution. In the vast majority of cases the distinction between psychogenic visual complaints and malingering is not made, and the term non-organic visual loss is used.

In this article we review the literature concerning NOVL, and provide up-todate guidelines on the most appropriate investigative and therapeutic approach to patients with unexplained visual symptoms.

\section{Loss of acuity}

NOVL is more common among younger age-groups and females. ${ }^{13-8}$ The most frequently reported non-organic visual complaints include a reduction of visual acuity with or without loss of field (36-80\%). ${ }^{39}$ Isolated visual field defects in the presence of normal acuity are less frequent at $14-20 \%,{ }^{39}$ and visual symptoms are binocular in the majority of cases $(50-80 \%))^{3}{ }^{510-12}$

There are no features of a clinical history that are pathognomonic of non-organic visual loss, but the onset of alleged visual loss is usually not typical of known pathology. It has been noted, however, that patients with psychogenic complaints are highly suggestible, whereas malingerers "try too hard" to convince the physician of their symptoms. ${ }^{13}$ A history of impending personal-injury litigation, although suggestive of visual symptoms feigned for secondary financial gain, is unhelpful because all patients must be examined as if they have disease until proven otherwise.

Establishing a diagnosis of NOVL is easier in those cases where markedly decreased vision is alleged as less quantitative tests are required. One such example, known as the "mirror test", involves the examiner rotating a mirror in front of the subject to induce a pursuit movement of the visually perceived image. ${ }^{14-16}$ The principle that optokinetic nystagmus, elicited with a drum or tape, indicates a visual acuity of $6 / 60$ or better, can also be used in these cases. ${ }^{14}{ }^{15}$ Although there are no clinical tests to distinguish between psychogenic and feigned visual loss, it has been noted that the malingerer is usually unable to sign his/her name whereas a truly blind person has no difficulty in performing this task. ${ }^{13}$ Simple tests of proprioception are also easily performed by the genuinely non-seeing patient but malingerers, believing the exercise to be vision dependent, are frequently incapable of bringing their index fingers together in front of their eyes. ${ }^{17}$ These techniques can be used for suspected monocular or binocular non-organic visual loss, but the good eye should be occluded in cases of monocular complaints (box).

If a patient claims bilateral total or near-total blindness, clinical observation is the most useful tool to the ophthalmologist. Findings not consistent with an organic aetiology under such circumstances include a smooth entrance into the consulting room, an intact menace reflex, flinching with increased illumination or a failure to direct the eyes towards their own hands during manual tasks. ${ }^{13}$ 
Table 1

\begin{tabular}{|c|c|c|}
\hline Test & Technique & Comments \\
\hline Fogging test & $\begin{array}{l}\text { Plus lenses of progressively increasing power are placed before } \\
\text { the 'unaffected' eye while the vision is tested (neither eye } \\
\text { occluded); eventually the 'unaffected' eye will be sufficiently } \\
\text { blurred for the examiner to be certain that the 'affected' eye is in } \\
\text { fact reading. }\end{array}$ & $\begin{array}{l}\text { Useful for alleged mild reductions in vision; only applicable when } \\
\text { reported visual symptoms are asymmetric }\end{array}$ \\
\hline Prism shift test & $\begin{array}{l}\text { A four dioptre base-out prism is placed in front of the eye with } \\
\text { alleged visual reduction while fixating a Snellen letter; if } \\
\text { movement is detected by the examiner, or diplopia } \\
\text { acknowledged by the patient, an acuity approximately equal to } \\
\text { the Snellen letter is confirmed. }\end{array}$ & $\begin{array}{l}\text { Useful for alleged mild reductions in vision; only applicable when } \\
\text { reported visual symptoms are asymmetric }\end{array}$ \\
\hline Polarizing lens tests & $\begin{array}{l}\text { The subject wears polarizing glasses, with one axis at } 90^{\circ} \text { and the } \\
\text { other at } 180^{\circ} \text {, while looking at projected letters which can be } \\
\text { selectively made visible to the right eye only, the left eye only or } \\
\text { to both eyes. The patient is therefore unaware which eye is being } \\
\text { tested. }\end{array}$ & $\begin{array}{l}\text { The acuity of each eye can be tested independently; monocular clues are } \\
\text { available; only applicable when reported visual symptoms are asymmetric }\end{array}$ \\
\hline Stereoscopic tests & $\begin{array}{l}\text { Appreciation of the various disparate images of the Titmus } \\
\text { stereo test indicates a degree of stereopsis that can be calculated } \\
\text { by referring to an accompanying chart; } 40 \mathrm{~s} \text { of arc of } \\
\text { stereo-acuity is equivalent to a Snellen acuity of } 6 / 6 \text { or better in } \\
\text { each eye. }\end{array}$ & $\begin{array}{l}\text { The acuity of both eyes can be tested simultaneously; poor stereoscopic } \\
\text { acuity is inconclusive }\end{array}$ \\
\hline Preferential looking & $\begin{array}{l}\text { The subject's fixation is observed while grating acuity cards of } \\
\text { varying stripe widths, and uniform grey targets, are presented. } \\
\text { Subjects with acuity sufficient to see the stripes will show a } \\
\text { fixation preference for the grating pattern. As the different stripe } \\
\text { widths correspond to their respective Snellen letters, the visual } \\
\text { acuity can be determined. }\end{array}$ & $\begin{array}{l}\text { This test can be used for alleged monocular or binocular reductions in } \\
\text { vision; this test tends to fail if complete blindness is claimed }\end{array}$ \\
\hline
\end{tabular}

The first step in any ophthalmic examination is testing of visual acuity. Physicians can become quite frustrated if they strongly suspect the vision to be better than the patient alleges, especially in the setting of a busy out-patient clinic. It is important that such sentiments, however justified, are not apparent to the patient. It is a good idea to postpone seeing such cases until the end of the clinic when one is less pressed for time.

Patients claiming mild to moderately reduced vision in one or both eyes present the greatest challenge to the physician. Several techniques designed to demonstrate that the patient has better vision than indicated by the subjective response have been described, and each test has its own merits and limitations. On the subject of these techniques, Sir Stewart Duke-Elder said ".... [they] will expose both simulated and functional conditions of a hysterical type, all of which are in essence trickery to match trickery, either conscious or subconscious". ${ }^{18}$ The tests described below can be performed in the out-patient department using commonly available materials and although it is not necessary to perform all of them to establish the non-organic nature of a visual complaint, the physician should be comfortable with a few techniques so that they can be performed smoothly without arousing the patient's suspicion (table).

THE FOGGING TEST

The most frequently used method is based on the principle of 'fogging'. ${ }^{13-16}$ First the ophthalmologist establishes which eye has better vision subjectively, and then places a weak plus spherical lens in front of that eye. Visual acuity is tested again (neither eye occluded) and the patient is encouraged to 'try really hard'. Gradually lenses of increasing strength are put before the 'better' eye, and the examiner retests the vision with each change of lens. When the optimum visual acuity attainable is demonstrated, the 'bad eye' is occluded and the patient is once again asked to read the acuity chart. The 'fogging' effect of the plus lenses will have reduced the visual acuity of the 'good eye' to such an extent that not even the top letter $(6 / 60)$ is visible to the patient, thus indicating that the patient was in fact reading with the 'bad eye'. The main limitation of the 'fogging' test rests on the fact that it can only be used when the alleged visual deficit is asymmetric.

PRISM SHIFT TEST

A prism in cross-section has an apex and a base, and light passing through a prism is refracted towards its base. The power of a prism is a measure of the deviation of a light ray traversing the prism, and is measured in prism diopters. Under normal circumstances, a prism placed before a seeing eye will displace the image and the subject will experience diplopia. The fixating eye will also make a small movement to ensure that the image still falls on the fovea. This principle can be used to assess the validity of a patient's visual symptoms.

A four dioptre base-out prism is placed in front of the 'affected' eye while the patient is fixating a Snellen letter. If movement is detected during the exercise, 
or diplopia acknowledged, than an acuity approximately equal to the Snellen letter is confirmed. ${ }^{15-16}$ This test is useful for alleged mild, but asymmetric, reductions in vision.

READING BAR

In this test the patient is instructed to read some printed matter at a distance of 14-16 inches while a vertical bar (eg, a tongue depressor) is placed 7 inches in front of the patient's face. The subject will be able to read continuously if the acuity of both eyes is good, whereas the vertical bar will block the better eye and interrupt reading if the fellow eye has poor vision. ${ }^{15}$

POLARIZING LENS TESTS

The patient wears polarizing glasses, with one axis at $90^{\circ}$ and the other at $180^{\circ}$, while looking at projected letters which are visible to one eye only, or to both eyes. Thus, as the patient reads the chart, the examiner can selectively 'occlude' either eye. The first few letters should be visible to both eyes so that the patient does not suspect trickery. Using this technique the ophthalmologist can establish the true acuity of each eye individually. ${ }^{15}{ }^{16}$ Disadvantages of tests using polarizing lenses include that their use is limited to alleged asymmetric visual impairment, and monocular clues are available.

STEREOSCOPIC TESTS

Sensory fusion is the process whereby differences between the image perceived by the right eye and the left eye are not appreciated, thus resulting in binocular single vision. Stereopsis, or binocular depth perception, is possible because of fusion of these disparate images. Stereopsis requires good vision in both eyes. Several techniques exist to assess binocular depth perception, and the Titmus stereo test is one of these. The Titmus test consists of a series of images, only some of which contain disparities and appear elevated when viewed through Polaroid glasses. The images are calibrated, and each represents a different level of stereopsis.

Stereoscopic acuity correlates well with Snellen acuity, and it has been shown that 40 seconds of arc of stereo-acuity is equivalent to no less than $6 / 6$ acuity in both eyes. ${ }^{19}$ Therefore, correct identification of 9 of 9 circles in the Titmus Stereo Test indicates normal vision bilaterally. The main advantage of stereoscopic tests is that the visual acuity of both eyes can be tested simultaneously, whereas its main limitation rests on the fact that poor stereoscopic acuity is inconclusive.

COLOURED LENSES AND CHARTS

This test is based on the principle that coloured glass will filter out complementary colours. The examiner can therefore identify the eye being used while testing the visual acuity by instructing the patient to read a chart with alternate red and green letters through red/green glasses. For example, if the red lens is placed before the 'bad' eye and the subject reads the entire chart we know the eye with allegedly poor vision is being used..$^{15}{ }^{16}$ The main disadvantages of red/green dissimilar image tests include the loss of one to two lines of Snellen acuity with the goggles, and they are only useful in cases where the alleged reduction in acuity is asymmetric.

PREFERENTIAL LOOKING

In this test the patient's fixation is observed while a uniform grey target and grating acuity cards of varying stripe widths are presented. ${ }^{20}$ The subject will show a fixation preference for the grating pattern if the visual acuity is sufficient to see the stripes. There are two main advantages of this test. First, an approximate level of acuity can be documented. Second, it can be used for alleged monocular or binocular reductions in vision. However, this test does tend to fail if complete blindness is reported. ${ }^{20}$

POTENTIAL ACUITY METER

The potential acuity meter, or interferometer, was designed to evaluate retinal visual acuity prior to cataract surgery so that patients could be furnished with a realistic visual prognosis pre-operatively. This instrument consists of a monochromatic light source which generates interference stripes posterior to the lens, and which are therefore not affected by lenticular opacities. Prior to the examination, the patient is told that the test is designed to circumvent the current eye problem and estimate what the vision would have been like had the injury or illness not occurred. The patient's recognition of the interference stripes generated by the interferometer is used as a measure of retinal acuity as each stripe width corresponds to a level of Snellen acuity. ${ }^{11}$ 
The advantages of the potential acuity meter include its ability to measure the visual acuity, and its use in monocular or binocular visual complaints.

NOVL should be suspected when a complete ophthalmic and neuroophthalmic assessment fails to identify pathology or pathologies which can account for the visual complaint. Ocular pathologies with subtle clinical signs should always be borne in mind in the differential diagnosis of unexplained visual symptoms and these include keratoconus, cone dystrophies, early Stagardt's disease, amblyopia, retrobulbar optic neuritis and optic nerve compression. ${ }^{132122}$ During the examination particular attention should be directed to pupillary reactions and the swinging light test. It should be noted, however, that organic and non-organic disease of the visual system often co-exist and previous investigators have reported ocular comorbidity in $25-53 \%$ of patients with NOVL. ${ }^{1023}$

\section{Loss of field}

Non-organic loss of visual field usually occurs in association with an alleged reduction in visual acuity. ${ }^{39}$ Several types of factitious fields have been reported, but concentric loss of peripheral vision with consequential 'tunnel vision' is the most frequently described. ${ }^{24}$ Suspicion is initially aroused at the ease with which the patient manoeuvres around objects upon entering the consulting room.

In cases of suspected non-organic loss of field, appropriate techniques include tangent screen testing, Goldmann perimetry and plotting of the visual field with both eyes open. Automated visual field analysis is inappropriate for several reasons. First, organic and non-organic defects cannot be distinguished using automated techniques. ${ }^{25-27}$ Second, the indices of reliability, including numbers of repetitions, false positives, false negatives and percentage of fixation losses, are similar for cases of field loss with or without physical basis and consequently the physician is presented with yet another 'abnormal' result. ${ }^{25}{ }^{27}$ Finally, malingerers fake field abnormalities which are more characteristic of classical neurological field defects than those seen in cases of genuine neurological disease. ${ }^{27}$

Psychogenic or feigned field defects remain unchanged in width when tangent screen testing is performed at varying distances while maintaining equivalence by adjusting target size, and are consequently known as 'tubular fields'. In the presence of true loss of field, the area of constricted field expands with increasing test distance. ${ }^{14}$

The most celebrated of the factitious fields in patients with NOVL is the spiral field. This is detected on Goldmann kinetic perimetry when the subject acknowledges the stimulus a little bit further out in each meridian when the first eye is being tested, regardless of whether the examination is proceeding clockwise or anti-clockwise.$^{17}$ On testing the second eye, a severely constricted field will be observed from the start. If the field of each eye is tested again on a separate occasion, but in the opposite order, the pattern will be reversed thus confirming the non-organic nature of the complaint.

A star-shaped field is also characteristic of psychogenic or feigned visual complaints, and is produced when the technician tests successive meridians from both sides before proceeding to the next one. ${ }^{28}$ However, it should be noted that spiral and star-shaped fields are actually the result of poor reproducibility and can therefore also be seen in association with fatigue. ${ }^{17}$ Other findings on kinetic perimetry which are suggestive of NOVL include non-physiologic overlap of isopters, variability in the pattern of field loss between successive examinations and poor reproducibility of the plotted field during a single session. ${ }^{28}$

Monocular hemianopias are also usually without physical basis, and are almost invariably associated with a complete hemianopia on binocular testing. ${ }^{14}{ }^{29}$ The non-organic nature of this field defect is revealed by finding a complete hemianopia on binocular testing rather than the expected small temporal crescent. ${ }^{29}$ Also, if two stimuli are presented to the 'blind' hemifield, the patient often reports that he/she can see the one closer to the 'good eye'. ${ }^{14}$

\section{Visual electrophysiology}

The International Society for Clinical Electrophysiology of Vision recommends that all patients with unexplained visual loss undergo standardised electroretinography, pattern electroretinography, and pattern appearance visually evoked cortical potentials (VECPs). ${ }^{30}$ Other forms of electrophysiological tests such as electro-oculography, bright electroretinography and flash VECPs are noncontributory in such cases and are therefore not indicated. ${ }^{30}$ 
VISUALLY EVOKED CORTICAL POTENTIALS

VECPs are thought to originate from the occipital cortex, and are recorded to investigate the integrity of the retinogeniculostriate pathway. ${ }^{31}$ However, in the context of unexplained visual symptoms the VECP has several limitations.

First, normal or near-normal cortical potentials in response to sinusoidal and checkerboard gratings have been reported in the presence of almost complete destruction of the occipital lobes, indicating that recordable electrical signals can be generated in the absence of sufficient striate cortex for vision. ${ }^{32}$

Second, subjects can voluntarily alter the pattern VECP and this is of particular importance in cases of malingering. ${ }^{33}$ Retinal blur induced by purposeful defocusing of the test stimulus may result in decreased amplitude and prolonged latency of the cortical response. Some subjects can completely obliterate their VECP response on instruction, whereas others can reduce its amplitude through volitional suppression. Eye movements and eccentric fixation can also result in abnormal VECP responses. Careful monitoring of subject cooperation is therefore crucial when recording visually evoked cortical potentials if the results are to be considered valid.

Finally, electrophysiological demonstration of a normal pathway between the retina and primary cortex is neither necessary nor sufficient to establish a diagnosis, ${ }^{32}$ as co-existing pathology of the visual system is in fact relatively common in patients with NOVL. ${ }^{10}{ }^{23}$

Despite the aforementioned limitations, VECP is a measure of pathway integrity and therefore remains a vital tool in the assessment of unexplained visual loss. ${ }^{16} 2333{ }^{34}$ However, like all electrophysiological investigations, visually evoked cortical potentials should always be interpreted within the context of the clinical findings and with full appreciations of the test's limitations.

PATTERN ELECTRORETINOGRAPHY

The pattern electroretinogram is a retinal response that can be evoked by viewing a temporally modulated pattern stimulus of constant total luminance (grating or checkerboard). A normal pattern electroretinogram response depends on the functional integrity of the retina and the optic nerve. The role of the pattern electroretinogram in unexplained visual loss is two-fold. First, it will detect photoreceptor dysfunction syndromes with subtle funduscopic signs. ${ }^{22}$ Second, a normal pattern electroretinogram response indicates that the image on the retina is well focussed and that fixation by the patient was adequate. Consequently, the simultaneous recording of the pattern electroretinogram and the VECP has been found to be particularly useful in cases of psychogenic and feigned visual symptoms. $^{35}$

\section{Psychophysical testing}

Colour vision testing is indicated in cases of unexplained visual reduction. Patients with cone dysfunction syndromes will often have ophthalmoscopically normal fundi, but colour discrimination losses and electroretinography abnormalities will be evident. ${ }^{21}{ }^{22}$

Dark-adaptometry, although not specifically indicated unless the patient reports night blindness, may be helpful as it can provide positive results which are unique to NOVL. ${ }^{36}$ The majority of patients with non-organic visual complaints exhibit an upward shift in threshold after prolonged testing (the 'exhaustion phenomenon'), monophasic dark adaptation and significantly raised absolute rod and cone thresholds. ${ }^{36}$ The 'exhaustion phenomenon' is said to be pathognomonic of NOVL. ${ }^{36}$

\section{Neuro-imaging}

A diagnosis of non-organic visual loss is made when the visual acuity is shown to be better than subjectively alleged, and where the functional integrity of the afferent visual pathway is confirmed by clinical examination with or without supplementary electrophysiological investigations. Neuro-imaging is indicated only in selected cases. Patients with reproducible field defects or suspected cortical blindness, in addition to those for whom normal vision cannot be restored during the course of the examination, should undergo computed tomography and/or magnetic resonance imaging.

\section{Aetiology/pathogenesis}

The pathogenesis of psychogenic visual complaints remains obscure, but it is assumed that higher cortical structures occupied with sensory awareness suffer from a disturbance. ${ }^{17}$ Conversion and somatization disorders, as well as 
hypochondriasis, have all been implicated in the process. ${ }^{17}$ A conversion reaction uses a physical symptom to express a psychological conflict. ${ }^{37}$ A classic example is that of the German corporal who became blind in his shooting eye after being ordered to execute Jews in 1941. Although he was relieved of that murderous task, the psychogenic visual defect lasted 52 years until he lost vision in the fellow eye. ${ }^{38}$ Hypochondriasis and somatization disorders usually involve several organ systems, and symptoms tend to be more chronic than in conversion reactions. $^{37}$

There is no clear consensus in the literature on whether or not a psychiatric evaluation is indicated in cases of non-organic visual loss. ${ }^{3172423}$ Of 42 patients with psychogenic or feigned visual complaints assessed by a psychiatrist in the series reported by Kathol et al, $22(52 \%)$ were found to have psychiatric syndromes. ${ }^{23}$ However, the authors reported that no patient benefited from psychotherapy and that the psychiatric condition did not contribute to the visual symptoms in many of these cases. ${ }^{23}$ Catalano et al described 23 children with NOVL for whom conflicts in the school or in the home were common, but only one had a psychiatric diagnosis. ${ }^{5}$ It is generally believed that non-organic visual complaints are, in many cases, a manifestation of suggestibility which is unlikely to affect normal daily living in an adverse way. ${ }^{39}$ We agree with previous workers that a diagnosis of NOVL is not in itself an indication for psychiatric referral, unless it is accompanied by other symptoms suggestive of a psychiatric disorder. ${ }^{39}$

The malingerer reports, but does not experience, his or her symptoms. The motivation for this pretence is known as secondary gain, and the most frequently described examples include compensation claims and avoidance of military service. ${ }^{10}{ }^{13}$ In cases of established NOVL it is not the role of the ophthalmologist to distinguish between psychogenic or feigned visual symptoms, but to manage the patient appropriately so that a rapid visual recovery ensues. However, ophthalmologists should be aware that clinical neuropsychologists can test the validity of a patient's symptoms using the forced-choice method, and this technique is often used in cases of personal injury litigation. ${ }^{40}$ The forced-choice technique is performed by asking the patient to make many successive two-alternative judgments regarding visual stimuli presented in a random order. If the complaint is valid, then by chance alone approximately $50 \%$ of choices will be correct. If the correct score falls significantly below chance expectation, the validity of the subject's symptoms are in question.

\section{Management}

To our knowledge, there are no published prospective treatment trials regarding the visual outcome of NOVL. Methods used in the past include psychoanalysis, ${ }^{41}$ sensory deprivation, ${ }^{15}$ confrontation, ${ }^{15}$ and placebo treatments. ${ }^{42}$ Religious cures are also well described. ${ }^{56}$ However, the cornerstone of management for psychogenic or feigned visual complaints remains reassurance by a concerned physician that there is no brain or eye disease, and psychotherapy is rarely indicated. ${ }^{9} 1323$ The natural history of non-organic visual loss should be explained and the expectation of complete visual recovery should be expressed, and an appointment for re-examination to document the improvement should be scheduled. Use of placebo treatments such as eye drops, orthoptic exercises or spectacles are not recommended and they will only serve to undermine the reassurance. ${ }^{13}$

In cases of childhood psychogenic or simulated visual symptoms, the ophthalmologist should take the time to talk to the parents alone. Parents should be reassured about the health of their child's eyes, and made aware of the excellent visual prognosis associated with NOVL in childhood. ${ }^{5}$ Terms such as 'pretending' should be avoided, and punitive measures should be actively discouraged. The possibility that the symptoms may be a somatic expression of stress should also be explained, and that improved communication between family members may accelerate the visual recovery. Children in whom the visual symptoms do persist often need psychological help, and therefore review appointments should be arranged. ${ }^{10}$ Although extremely rare, the possibility of physical or sexual abuse should be considered in all cases of childhood non-organic visual loss. ${ }^{10}$

\section{Visual prognosis}

Using the therapeutic approach described above, between $45 \%$ and $78 \%$ experience complete resolution of all visual symptoms. ${ }^{135234344}$ Good prognostic indicators include young age and absence of any associated psychiatric disease. ${ }^{34}$ Of those with a poor visual recovery, however, living incapacity can sometimes result. In Kathol's series of 42 patients, eight (19\%) considered 
themselves to be visually disabled at an average follow-up of four years. ${ }^{23} \mathrm{How}-$ ever, few of these were suffering socially or economically as a consequence of their visual problems, although one patient was studying in a programme financed by a school for the blind. ${ }^{23}$

Prospective multicentre studies investigating the visual outcome of nonorganic visual loss and the factors that influence it are needed if the variability in visual recovery following comparable regimes of reassurance is to be explained.

\section{Conclusion}

Non-organic visual loss remains a clinical diagnosis which is made when the physician demonstrates that the visual acuity is better than subjectively alleged, and fails to find pathology of the visual system to account for the patient's complaints. Electrophysiological investigations of the visual pathways are recommended in all cases.

The mainstay of management is reassurance that the brain and eyes are free of disease, and that a full visual recovery is expected. A diagnosis of non-organic visual loss is not an indication for psychiatric referral.

Although the visual prognosis is excellent in the majority of cases, improvement may be slow and incomplete in a small proportion of patients. Studies designed to identify the factors that influence the visual outcome in cases of NOVL are needed if we are to recognise and target those cases at risk of a poor visual recovery at an earlier stage.

1 Kathol RG, Cox TA, Corbett JJ, Thompson HS, Clancy J. Functional visual loss: I A true psychiatric disorder? Psychol Med 1983;13:307-14.

2 Mace CJ, Trimble MR. 'Hysteria', 'functional' or 'psychogenic'? A survey of British neurologists' preferences. I R Soc Med 1991;84:471-5.

3 Sletteberg O, Bertelsen T, Hovding G. The prognosis of patients with hysterical visual impairment. Acta Ophthalmol 1989;67:159-63.

4 Hafeiz HB. Hysterical conversion: a prognostic study. Br f Psychiatry 1980;136:548-51.

5 Catalano RA, Simon JW, Krohel GB, Rosenber

PN. Functional visual loss in children. Ophthalmology 1986;93:385-90.

6 Yasuna ER. Hysterical amblyopia in children. Am f Dis Child 1963;106:558-63.

Am f Dis Child 1963;106:558-63.
7 Krill AE, Newell FW. The diagnosis of ocula 7 Krill AE, Newell FW. The diagnosis of ocular conversion reaction involving vis

Arch Ophthalmol 1968;79:254-61.
8 Rada RT, Meyer CG, Krill AE. Visual conversion reaction in children. I Diagnosis. Psychosomatics 1969;10:23-8.

9 Barris MC, Kaufman DI, Barberio D. Visual impairment in hysteria. Invest Ophthalmol Vis Sci 1992;33:1227-32.

10 Keltner JL, May WN, Johsnon CA, Post RB. The California syndrome. Functional visual complaints with potential economic impact. Ophthalmology 1985;92:427-35.

11 Grignolo FM, Moscone F, Carenini-Boles A. Assessment of malingerer's visual acuity by Lotmar's visometer test. Ann Ophthalmol 1988;20: mar's visc

12 Bobak P, Khanna P, Goodwin J, Brigell M. Pattern visual evoked potentials in cases of ambiguous acuity loss. Doc Ophthalmol 1993;85:18592.

13 Thompson HS. Functional visual loss. Am $\mathscr{f}$ Ophthalmol 1985;100:209-13.

14 Smith CH, Beck RW, Mills RP. Functional disease in neuro-ophthalmlogy. Neurol Clin. 1983, 1:955-71.

15 Kramer KK, LaPiana FG, Appleton B. Ocular malingering and hysteria: diagnosis and management. Surv Ophthalmol 1979/80;24:89-96.

16 Miller BW. A review of practical tests for ocula malingering and hysteria. Surv Ophthalmol 1973;17:241-6.
17 Weller W, Wiedemann P. Hysterical symptoms in ophthalmolgy. Doc Ophthalmol 1989;73:1-33.

18 Duke-Elder S. Ophthalmic optics and refraction. London: Henry Kimpton, 1970; pp 490-9.

19 Donzis PB, Rappazzo JA, Burde RM, Gordon M. Effect of binocular variations of Snellen's visual acuity of Titmus stereoacuity. Arch visual acuity of Titmus st 101:930-2.

20 Fahle M, Mohn G. Assessment of visual function in suspected ocular malingering. $\mathrm{Br} F$ Ophthalmol 1989;73:651-4.

21 Zervas JP, Smith JL. Neuro-ophthalmic presentation of cone dysfunction syndromes in the adult. I Clin Neuroophthalmol 1987;7:202-18.

22 Rowe SE, Trobe JD, Sieving PA. Idiopathic photoreceptor dysfunction causes unexplained visual acuity loss in later adulthood. Ophthalmology 1990;97:1632-7.

23 Kathol RG, Cox TA, Corbett JJ, Thompson HS Functional visual loss. Follow-up of 42 cases. Arch Ophthalmol 1983;101:729-35.

24 Newman NJ. Neuro-ophthalmology and psychiatry. Gen Hosp Psychiatry 1993;15:102-114.

25 Smith TJ, Baker RS. Perimetric findings in functional disorders using automated techniques. Ophthalmology 1987;94:1562-1566.

26 Thompson JC, Kosmorsky GS, Ellis BD. Field of dreamers and dreamed-up fields. Functional and fake perimetry.Ophthalmology 1996;103: $117-125$

27 Stewart JFG. Automated perimetry and malingerers. Can the Humphrey be outwitted? Ophthalmology 1995;102:27-32.

28 Ohkubo H. Visual field in hysteria- reliability of visual field by Goldmann perimetry. Doc Ophthalmol 1989;71:61-67.

29 Keane JR. Hysterical hemianopia. The 'missing half' field defect. Arch Ophthalmol 1979;97:865866

30 Galloway NR, Odom JV, Anastasi M, et al for the Working Party of the International Society for Clinical Electrophysiology of Vision. Visual electrodiagnostics: $A$ guide to procedures. Nottingham: ISCEV Publications, 1997; pp 2-5

31 Halliday AM, Michael WF. Changes in patternevoked responses in man associated with the vertical and horizontal meridians of the visual field. F Physiol 1970;208:499-513.

32 Spehlmann R, Gross RA, Ho SU, Leestma JE, Norcross KA. Visual evoked potentials and postmortem findings in a case of cortical blindness. Ann Neurol 1977;2:531-4.

33 Howard JE, Dorfman LJ. Evoked potentials in hysteria and malingering. If Clin Neurophysiol 1986;3:39-49.

34 MacCana F, Bhargava SK, Kulikowski JJ. Flash and pattern VEPs: examples of cases of hysterical amblyopia and provoked visual impairment (Uhtoff's sign). Ophthalmic Physiol Opt 1983;3: 55-60.

35 Rover L, Bach M. Pattern electroretinogram plus visual evoked potential: a decisive test in patients suspected of malingering. Doc Ophthalmol 1987;66:245-51.

36 Krill AE. Retinal functional studies in hysterical amblyopia. Am f Ophthalmol 1967;53:230-7.

37 American Psychiatric Association (APA). Diagnostic and statistical manual of mental disorders. (DSM-III-R). Washington: APA, 1987.

38 Kluxen G. Psychogenic blindness caused by a command to kill. Psychother Psychosom Med Psychol 1995;45:131-3.

39 Kathol RG, Cox TA, Corbett JJ, Thompson HS, Clancy J. Functional visual loss: II Psychiatric aspects in 42 patients followed for 4 years. Psychol Med 1983;13:315-24.

40 Hiscock M, Hiscock CK. Refining the forcedchoice method for the detection of malingering. f Clin Exp Neuropsychol 1989;11:967-74.

41 Wilkins LG, Field PB. Helpless under attack: hypnotic abreaction in hysterical loss of vision. Am 7 Clin Hypnosis 1968;10:271-5.

42 Yasuna ER. Hysterical amblyopia in children and young adults. Arch Ophthalmol 1951;45:70-

43 Barris MC, Kaufman DI, Barberio D. Visual impairment in hysteria. Doc Ophthalmol 1992; 82:369-82.

44 Rada RT, Krill AE, Meyer GG, Armstrong D. Visual conversion reaction in children. II Follow-up. Psychosomatics 1973;14:271-6. 